\title{
Cytology of Feather Pulp Lesions from Marek's Disease (MD) Virus-Infected Chickens and Its Application for Diagnosis and Prediction of MD
}

\author{
Kyoung-Oh CHO, Nam-Yong PARK ${ }^{1)}$, Daiji ENDOH ${ }^{2)}$, Kazuhiko OHASHI, Chihiro SUGIMOTO, \\ Chitoshi ITAKURA ${ }^{3)}$ and Misao ONUMA \\ Laboratories of Infectious Diseases and ${ }^{2}$ Radiation Biology, Graduate School of Veterinary Medicine, Hokkaido University, Sapporo \\ 060-0818, Japan, I)Department of Veterinary Pathology, College of Veterinary Medicine, Chonnam National University, Kwang-Ju, \\ Korea,${ }^{4}$ Brain Science Institute, Institute of Physical and Chemical Research, Hirosawa 2-1, Wako-Shi, Saitama 351-01, Japan
}

(Received 29 January 1998/Accepted 31 March 1998)

ABSTRACT. Cytological changes of feather pulp lesions (FPL) sampled chronologically from the same specific-pathogen free chickens inoculated with Marek's disease virus serotype 1 (MDV) were examined, comparing with their histological changes. The birds having Marek's disease (MD) lymphomas or nerve lesions exhibited the characteristic lesion changes on the cytological smears of FPL; the initial non-suppurative inflammatory to the late lymphomatous FPL. The birds having neither the MD visceral lymphomas nor the nerve lesions manifested only non-suppurative inflammatory FPL on the cytological smears throughout the experimental periods. Histological evaluation of FPL sampled from the same birds confirmed as above mentioned cytological results. From these results, the cytological evaluation of FPL proved to be an effective diagnostic and prognostic tool in foreseeing MD incidence. - KEY wORDS: cytology, diagnostic and prognostic tool, feather pulp lesion, Marek's disease.

J. Vet. Med.Sci. 60(7): 843-847, 1998

Marek's disease virus (MDV) is a cell-associated herpesvirus. After a brief cytolytic infection of several days' duration in $\mathrm{MDV}$ infection, mainly $\mathrm{CD} 3{ }^{+} \mathrm{CD} 4{ }^{+} \mathrm{CD} 8{ }^{-}$ lymphomas develop at many organs and tissues including the viscera, skin, and peripheral nerves as well as feather pulp $[2,4,10,12]$.

The feather pulp forms a core inside the feather tube [5]. Three types of feather pulp lesions (FPL) associated with Marek's disease (MD) are described [10]: R1-type is characterized by variable infiltration of mainly small lymphocytes. R2-type is edema and infiltration of plasma cells, small lymphocytes and heterophils. T-type is manifested by proliferation of mainly tumorous lymphoblasts.

The FPL are reported as an important source for diagnosing the MD since the incidence of MD has a close relationship to the change of FPL $[4,10]$. The change of R1-type to T-type in the FPL was observed in the chickens showing visceral or skin MD lymphoma. On the other hand, the change of R1-type to R2-type in the FPL was seen in the chickens recovered from MD. Although histopathological changes of feather pulp have been described by many workers, there are no detailed reports on the cytology of FPL. One of the reasons is that a hard, firm and gelatinous feather pulp gave rise to difficulty of the preparation of smears [7].

Clinical pathology has played a major role in the diagnosis of human and domestic animal diseases. The importance and accuracy of cytologic examination as a diagnostic tool has been studied and reviewed in the human and veterinary literature $[1,3,5,6,8,9,11]$.

This paper describes the cytological variants of FPL recognized in chickens inoculated experimentally with $\mathrm{Md} /$
$5 \mathrm{MDV}$ which were compared with the histopathological changes of FPL and discusses the application of cytology of FPL for the diagnosis and predilection of MD.

\section{MATERIALS AND METHODS}

Animals: Specific-pathogen-free White Leghorn chicks were used. The birds were housed in isolation room and given food and water ad libitum.

Virus: A very virulent $\mathrm{MDV}, \mathrm{MD} / 5$, is maintained in our laboratory. The virus was propagated in chicken embryo fibroblasts, and its infective titer was determined before use.

Experimental design and pathological examination: Oneday-old chicks were allotted randomly into two groups (A and B) of 40 and 20 birds each and housed separately. Birds in group A were inoculated intra-abdominally with 7200 focus-forming units (FFU) of $\mathrm{Md} / 5$ at 1 week of age. Birds in group B served as controls. Clinical signs were recorded daily in both groups.

The sampling procedure of feathers including skin specimens for pathological examination was the same as described by Cho et al. [4]. Briefly, three immature growing feathers with their associated skin tissues per bird were sampled from the cervical, dorsopelvic, and femoral tracts each. These tissue samples were fixed in Bouin's solution, processed routinely, embedded horizontally in paraffin wax, and stained with hematoxylin and eosin (HE). This was performed at 1 -week intervals from 1 to 9 weeks postinoculation (pi) in both groups. Experiment was terminated when the birds were 10 weeks old.

All birds that died or were killed in both experiments were immediately necropsied. Samples from almost all feather tracts, visceral organs (liver, lung, heart, kidney, 
proventriculus, intestine, testis, ovary and pancreas), and nerve tissues (brachial and sciatic nerve plexuses) were collected, fixed in $10 \%$ buffered formalin, and embedded in paraffin wax.

Cytological examination: Another three feathers were plucked from the different feather tracts of the same bird as above mentioned. The feather pulps were removed and minced on the clean glass microscope slide with sharp shaving knife. A second glass slide was placed across over the sample, and the pulp was allowed to spread between the two slides while they were pulled horizontally apart with gentle digital pressure. Smears were air dried and stained immediately by a modified quick Wright's stain, Diff Quik (Harleco Co., Gibbstown, NG, U.S.A.). Briefly, the smears were dipped five times each in fixation solution, solution $\mathrm{A}$ and solution B. After then, the slides were washed gently with tap water and distilled water, air dried and mounted through xylene.

\section{RESULTS}

The histological incidences of MD visceral lymphomas and nerve lesions are shown in Tables 1 and 2. MD visceral lymphomas (Fig. 1) and nerve lesions were observed in 26 $(65 \%)$ and $24(60 \%)$ birds, respectively, in group A at the end of the experiment. Five birds $(12.5 \%)$ in group A had no MD visceral lymphomas and nerve lesions. During the experimental period, six birds in group A died of severe cachexia and visceral lymphomas or severe paralysis of one or more of the extremities. Control birds in group B showed neither the MD visceral lymphomas nor the nerve lesions.

Cytological changes of feather pulp lesions (FPL) sampled chronologically: The birds having MD visceral tumors or nerve lesions showed chronologically distinctive changes on the cytological smears of FPL. The FPL sampled at 1 and 2 weeks pi were constantly mild cell aggregates consisting mainly of a few small lymphocytes. There was a progressive increase in the number of lymphoid cells in the FPL with time pi. At 3 weeks pi, the number of cell aggregates in the FPL was considerable (about 10 to 30 cells in each aggregate) and consisted mainly of small lymphocytes with few lymphoblasts (Fig. 2a). At 4 and 6 weeks pi, the FPL developed to moderate foci in size and comprised almost equal mixture of small lymphocytes and lymphoblasts. The FPL at 7 to 9 weeks pi were manifested by large lymphoid aggregate composed mainly of lymphoblasts with some small lymphocytes (Fig. 3a).

In the birds showing neither the visceral tumors nor the nerve lesions in group A, FPL were different from those described above. FPL sampled at 1 to 3 weeks pi exhibited mild small lymphocyte infiltration. With time pi, however, the number of the plasma cells increased gradually and then they were the most prominent cells in the FPL sampled at 7 to 9 weeks pi (Fig. 4a).

Cytological characteristics of infiltrating cells on smears of FPL: The small lymphocytes in the FPL sampled at initial time had very scant, blue cytoplasm and small round nuclei with densely aggregated smudged chromatin (Fig. 2a). Lymphoblasts observed mainly in lymphomatous FPL at late time were highly pleomorphic and had moderate to abundant amount of bluish cytoplasm and medium to large nuclei with round to ovoid profiles. Their nuclei had

Table 1. Incidence of visceral lymphomas and nerve lesions in MDV inoculated and control groups

\begin{tabular}{ccccc}
\hline Group & Treatment & $\begin{array}{c}\text { No. of } \\
\text { birds }\end{array}$ & $\begin{array}{c}\text { Visceral } \\
\text { lymphomas }\end{array}$ & $\begin{array}{c}\text { Nerve } \\
\text { lesions }\end{array}$ \\
\hline A & $\begin{array}{c}\text { MDV } \\
\text { inoculated } \\
\text { Control }\end{array}$ & 40 & 26 & 24 \\
B & 20 & 0 & 0 \\
\hline
\end{tabular}

Table 2. Cytological changes of feather pulp lesions (FPL) from chickens infected with MDV

\begin{tabular}{lll}
\hline & \multicolumn{2}{c}{ Cytological changes of FPL } \\
\cline { 2 - 3 } Phase (weeks pi $\left.{ }^{\text {a) }}\right)$ & Chickens with MD lesions ${ }^{\mathrm{c})}$ & Chickens without MD lesions \\
\hline I $\quad$ (1-3 weeks pi) & Mainly small lymphocytes & Mainly small lymphocytes \\
II (4-6 weeks pi) & $\begin{array}{l}\text { A mixture of lymphoblasts } \\
\text { and small lymphocytes }\end{array}$ & $\begin{array}{l}\text { A mixture of plasma cells } \\
\text { and small lymphocytes }\end{array}$ \\
III (7-9 weeks pi) & $\begin{array}{l}\text { Mainly lymphoblasts with } \\
\text { some small lymphocytes }\end{array}$ & $\begin{array}{l}\text { Mainly plasma cells with } \\
\text { some small lymphocytes }\end{array}$ \\
& $\begin{array}{l}\text { V, N, or V+N } \\
\text { No. of birds }\end{array}$ & $\begin{array}{l}\text { No lesions observed in } \\
\text { viscera and nerves }\end{array}$ \\
examined & having N, 15 having V+N) & 5 birds \\
\hline
\end{tabular}

a) pi: post-inoculation. b) V: MD visceral lymphomas observed. N: MD peripheral nerve lesions observed. $\mathrm{V}+\mathrm{N}$ : MD visceral lymphomas and nerve lesions observed. $\mathrm{c}$ ) MD-visceral lymphomas and peripheral nerve lesions 
clumped or coarse chromatin and one or two large prominent nucleoli (Fig. 3a). Plasma cells found mainly in the late FPL of birds showing neither the MD lymphomas nor the nerve lesions had eccentric round or ovoid nuclei with coarse chromatin and abundant blue cytoplasm (Fig. 4a). Mesenchymal cells in the feather pulp had abundant pale blue cytoplasm and large round to ovoid nuclei with coarse to clumped chromatin without nucleoli (Fig. 3a).

Histological changes of FPL sampled chronologically:

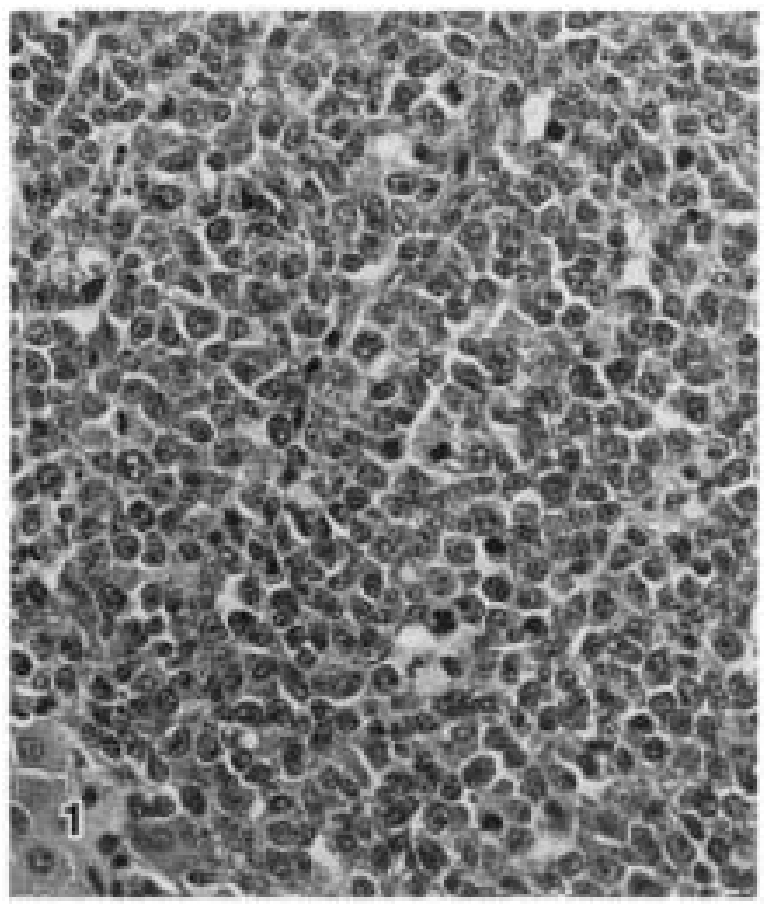

Fig. 1. Liver. Diffuse infiltration of tumorous lymphoblasts. At 9 weeks pi. HE stain. $\times 440$.
Cytological changes of FPL were compared to histological results of FPL sampled from the same birds as above mentioned. Histological changes of FPL sampled chronologically from the same birds were identical to cytological results of FPL as described above. Briefly, the initial FPL from birds exhibiting MD visceral lymphomas or nerve lesions were characterized by mild lymphoid cell aggregates consisting mainly of small lymphocytes (Fig. 2b). With time pi, the FPL showed expansive infiltration of lymphoid cells and finally consisted mainly of tumorous lymphoblasts in the late lesions (Fig. 3b). The birds with neither the MD visceral lymphomas nor the nerve lesions revealed distinctive lesion changes in feather pulp; the initial FPL consisted of a few small lymphocytes and the late FPL were composed mainly of plasma cells (Fig. 4b).

The incidence of MD FPL appearing on the cytological and histological slides was almost same.

\section{DISCUSSION}

The cytological changes on the smears of FPL in the present study were closely correlated with the incidences of MD visceral lymphomas or nerve lesions. The birds having MD visceral lymphomas or nerve lesions revealed the initial non-suppurative inflammatory to the late lymphomatous FPL in cytology. On the other hand, the birds showing neither the MD visceral lymphomas nor the nerve lesions manifested the initial inflammatory to the late inflammatory FPL in cytology. Histological observation of FPL sampled from the same birds at the same time confirmed the cytological changes of FPL. From these results, the cytological changes of FPL reflected the MD visceral lesions and it implied that the feather pulp was a suitable material for diagnosis of MD in the present study.

Cytologic samples can be collected quickly, easily and

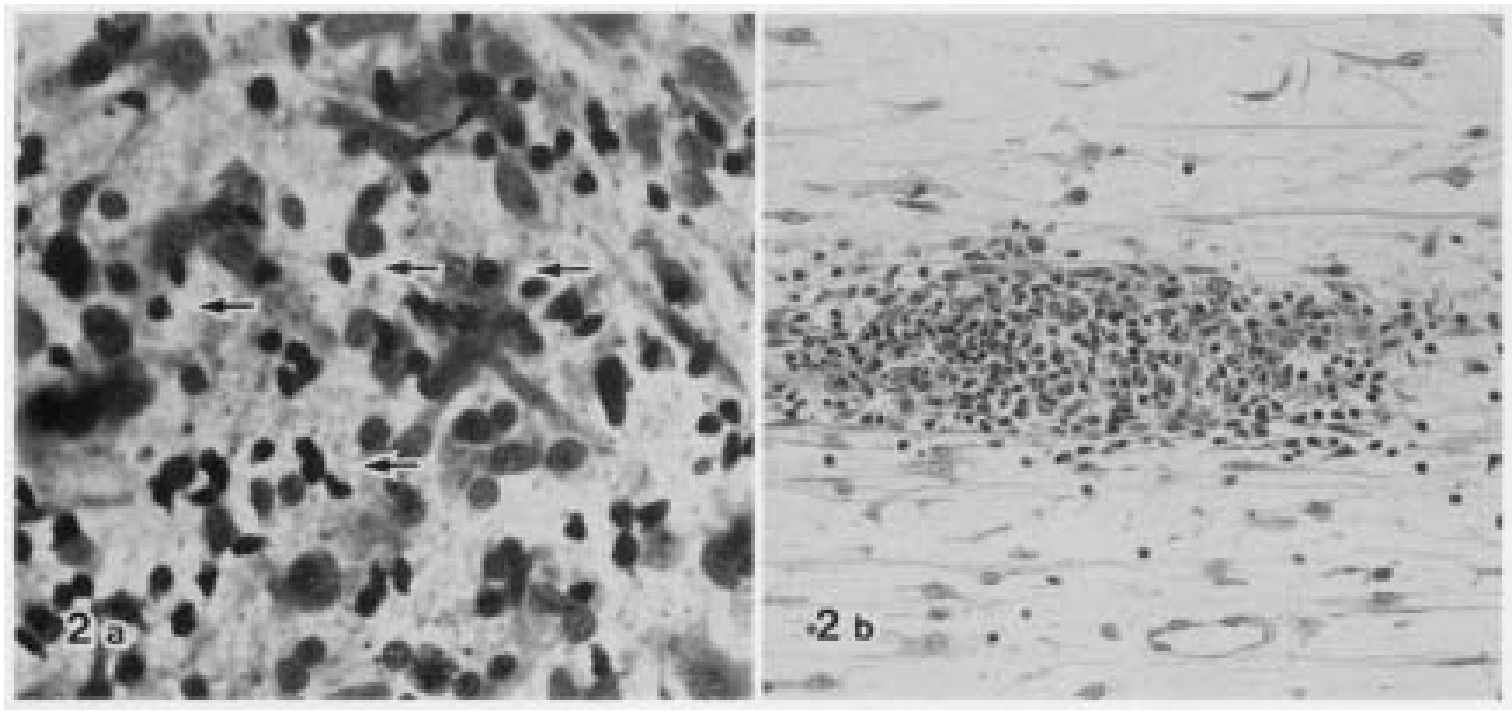

Fig. 2. a) Smear of feather pulp lesions sampled at 3 weeks pi, consisting mainly of small lymphocytes (arrows). Diff Quik stain. $\times 440$. b) Lymphoid cell aggregate composed mainly of small lymphocytes in feather pulp sampled at 2 weeks pi. HE stain. $\times 260$. 


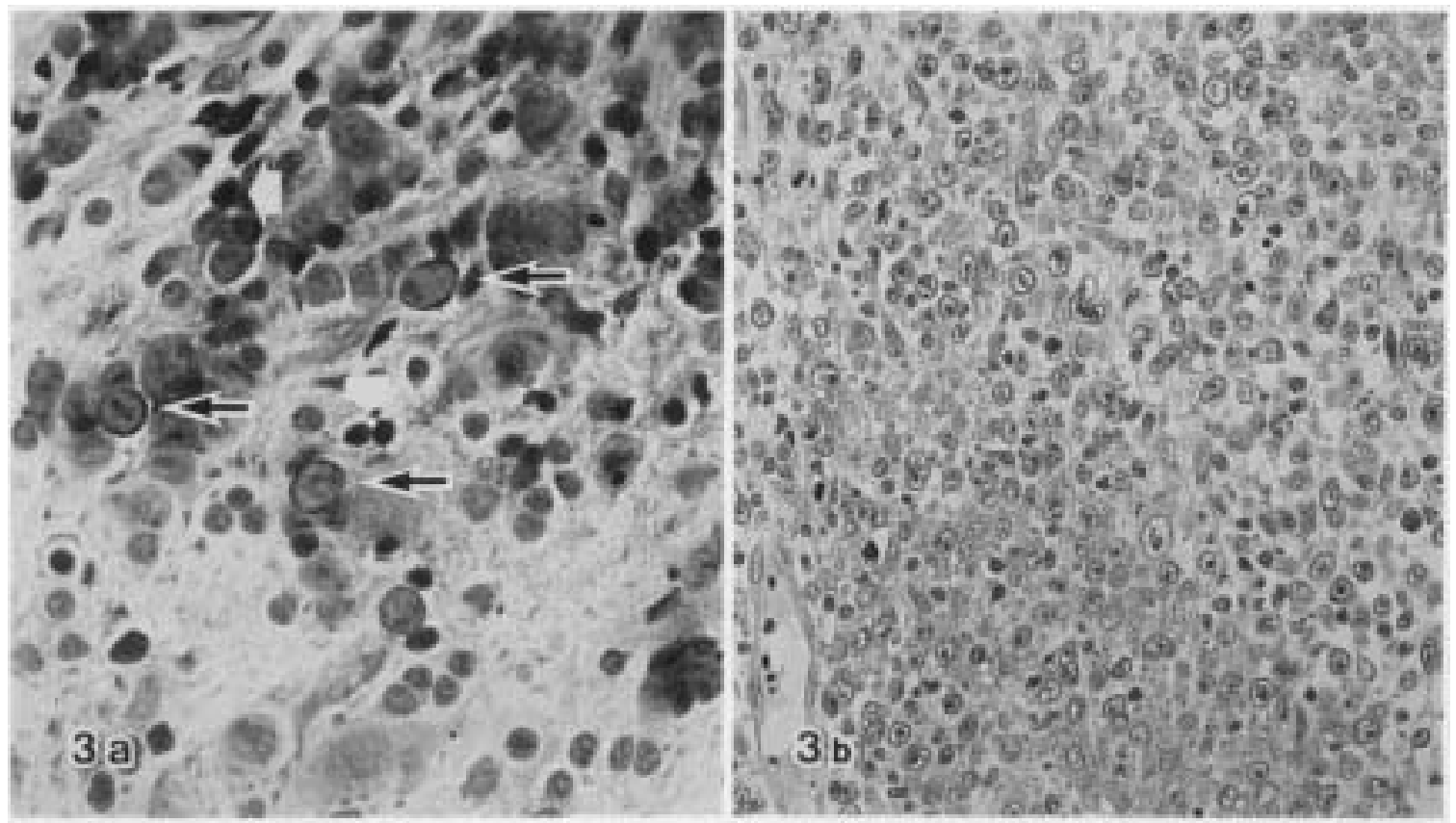

Fig. 3. a) Smear of feather pulp lesions sampled at 8 weeks pi, consisting mainly of tumorous lymphoblasts (arrows) with some small lymphocytes (thick arrows). Diff Quik stain. $\times 440$. b) Diffuse infiltration of tumorous lymphoblasts in a feather pulp sampled at 9 weeks pi. HE stain. $\times 330$.

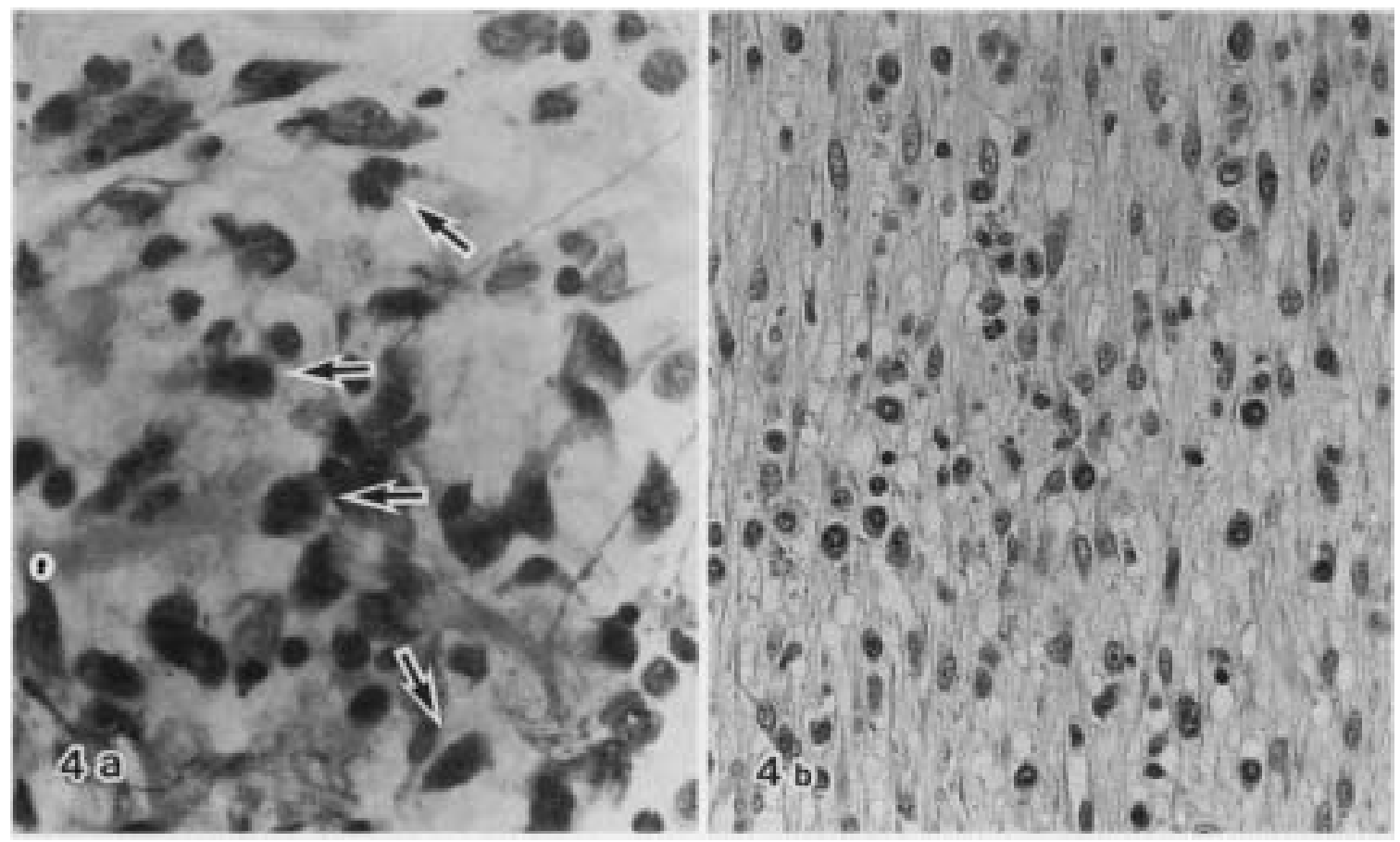

Fig. 4. a) Smear of feather pulp lesions sampled at 7 weeks pi, characterized by many plasma cell infiltration (arrows). Diff Quik stain. $\times 530$. b) Many plasma cell infiltration in feather pulp sampled at 7 weeks pi. HE stain. $\times 440$.

inexpensively, with little or no risk to the patient $[3,6,8$, $9,11]$. Evaluation of cellular responses can provide the veterinarian with a diagnostic aid in the development of a presumptive or definitive diagnosis $[3,6]$. To our knowl- edge, besides, the cytological changes of FPL in MD have not been reported. The present results provide the data for evaluation of cellular responses in cytological changes of FPL and may fulfill the above mentioned conditions. 
Cytologically, non-suppurative FPL in the present study were observed at initial time in both chickens having MD lesions or no in the viscera and peripheral nerves. This lesion is compatible with R1-type FPL described by Moriguchi et al. [10]. Cytologically, lymphomatous lesion in the present experiment is similar to that of T-type FPL which were observed in chickens having MD visceral lymphomas [10]. Moreover, the late inflammatory lesion consisting mainly of plasma cells in chickens without MD lesions is compatible with R2-type FPL which were observed in healthy chickens survived from MD. Moriguchi et al. [10] described two patterns of lesion development in feather pulp; R1-type to T-type and R1-type to R2-type, with the former progression occurring in birds which exhibited development of lymphoma in viscera. The latter progression was seen in birds that survived from MD. Therefore, these histological evaluation of FPL has been thought useful diagnostic tool of MD. Comparing with this histological evalulation, advantages to use the smear cytology for FPL may be its easiness as well as inexpensiveness and possible application for immunohistochemistry which is not suitable for formalinfixed and paraffin-embedded tissues.

Feather pulp is a homogenous, gelatinous substance that gives the entire pulp a firm, tough and elastic consistency [7]. Therefore, preparation of smears for cytologic evaluation of feather pulp was very difficult. In the present study, cytological preparation became easy and quickly, because feather pulp was minced by sharp knife for the smear preparation.

ACKNOWLEDGMENT. The authors thank Dr. K. Ochiai (Hokkaido University) for revision of Japanese abstract.

\section{REFERENCES}

1. Bottles, K., Miller, T. R., Cohen, M. B. and Ljung, B. M. 1986. Fine needle aspiration biopsy. Has its time come? Am. J. Med. 81: 525-531.

2. Calnek, B. W. and Witter, R. L. 1991. Marek's disease. pp. 342-385. In: Diseases of Poultry, 9th ed. (Calnek, B. W. ed.), Iowa State University Press, Ames.

3. Campbell, T. W. 1988. Avian hematology and cytology. 1st ed., Iowa State University Press, Ames.

4. Cho, K.-O., Mubarak, M., Kimura, T., Ochiai, K. and Itakura, C. 1996. Sequential skin lesions in chickens experimentally infected with Marek's disease virus. Avian Pathol. 25: 325343.

5. Cochand-Priollet, B., Chagnon, S., Ferrand, J., Blery, M., Hoang, C. and Galian, A. 1987. Comparison of cytologic examination of smears and histologic examination of tissue cores obtained by fine needle aspiration biopsy of the liver. Acta. Ctyol. 31: 476-480.

6. Cowell, R. L. and Tyler, R. D. 1989. Diagnostic cytology of the dog and cat. 1st ed., American Veterinary Publications, California.

7. Hodges, R. D. 1974. The histology of the fowl, 1st ed., Academic Press, New York.

8. Meyer, D. J. and Franks, P. 1986. Clinical cytology. Part 1: Management of tissue specimens. Mod. Vet. Pract. 67: 255259.

9. Mills, J. N. and Griffiths, G. L. 1984. The accuracy of clinical diagnoses by fine-needle aspiration cytology. Aust. Vet.J. 61: 269-271.

10. Moriguchi, R., Fujimoto, Y. and Izawa, H. 1982. Chronological observations of feather pulp lesions in chickens inoculated with Marek's disease virus. Avian Dis. 26: 375-388.

11. O'Rourke, L. G. 1983. Cytologic technics. Mod. Vet. Pract. 64: 185-189.

12. Schat, K. A., Chen, C. L., Calnek, B. W. and Char, D. 1991. Transformation of T-lymphocyte subsets by Marek's disease herpesvirus. J. Virol. 65: 1408-1413. 